to a higher level of debate about ideologies of simplicity and the "regular panics around 'dumbing down' of culture in general".

Leo Lentz

K. Rayner, B. Miller, C.M. Rotello (2008). Eye movements when looking at print advertisements: The goal of the viewer matters. Applied Cognitive Psychology 22 (5), 697-707.

How do people process print advertisements consisting of a picture as well as of text? This was investigated in an eye movement study in which subjects were given a number of ads to rate. Their task was varied systematically: half were asked to rate how much they liked each ad, while the other half were asked to rate the effectiveness of the ad. The results show several interesting mechanisms behind ad processing. First of all, data suggest that readers do not switch much between text and picture, but 'either sample the pictorial information and then use the text for confirmation, or they read the text and then sample the pictorial information for confirmation'. Second, subjects in this study tended to focus more on the picture part of the ad than on the text, but there were differences between different ads. In car and skin care ads, for example, the amount of time spent on text and picture was about equal, but this may have been caused by the fact that these ads just contained more text than the other ads in the set. Last, despite of what the title of the article might suggest, the two different rating tasks did not yield different looking time behaviors. However, the results of the present study do differ in comparison to a previous study (Rayner et al. 2001) in which subjects who were asked to decide whether they wanted to buy a product spent more time reading the text portion of the ad than looking at the picture.

Bregje Holleman

\section{Survey Design}

M.P. Couper, F.G. Conrad, R. Tourangeau (2007). Visual context effects in Web surveys. In: Public Opinion Quarterly 71 (4), 623-634.

Most people find surveys quite boring. Yet, they are an important means of obtaining information about political opinions, buying behaviors and attitudes. Survey designers sometimes try to make answering survey questions more fun by adding images to their (web) surveys. These images are not functional to the content of the survey: they do not offer an illustration of objects or products to accompany a description, or explain a procedure or task, but are included only to motivate or entertain the respondent, or for aesthetic reasons. As such, the images should not affect the content of people's responses, but just increase participation or reduce break offs. The assumption that responses are not influenced by the content of a picture was tested by including a picture of a healthy woman exercising versus a sick woman in a hospital bed presented close to a survey question in which respondents were asked to rate their own health.

The results show a consistent contrast effect: respondents seeing the picture of the sick woman report to be healthier than those exposed to the picture of the sporty woman. In one experiment, the effect seemed to disappear when the image was in the header, not ostensibly linked to the survey questions (suggesting 'banner blindness'), but in a subsequent experiment it did not matter whether the picture was in the survey or in the banner to the survey, nor did the size of the effect vary significantly 
with the size of the picture. The conclusion is that survey designers should be careful including images in web surveys, especially when their content is relevant to the content of questions in the survey.

Bregje Holleman 